\title{
Difficulties in Diagnosing Food-Borne Botulism
}

\author{
Nina Forss ${ }^{a, e} \quad$ Raimo Ramstad ${ }^{b, e}$ Tom Bäcklund $^{c}$ \\ Miia Lindström ${ }^{f} \quad$ Elina Kolho ${ }^{d}$ \\ Departments of ${ }^{a}$ Neurology, ${ }^{b}$ Clinical Neurophysiology, ${ }^{c}$ Internal Medicine and \\ ${ }^{d}$ Infectious Diseases, Helsinki University Central Hospital, and Departments of \\ ${ }^{\mathrm{e}}$ Clinical Neurosciences and ${ }^{\mathrm{f}}$ Food Hygiene and Environmental Health, \\ University of Helsinki, Helsinki, Finland
}

\section{Key Words}

Botulism · Bulbar palsy $\cdot$ Misdiagnosis

\begin{abstract}
Botulism is a muscle-paralyzing disease caused by neurotoxins (types A-G) produced by the bacteria Clostridium botulinum. Symptoms of food-borne botulism most commonly appear 12-36 $\mathrm{h}$ after eating contaminated food, but the earliest neurological symptoms may in some cases start abruptly. Here, we report the cases of two patients with food-borne botulism who were admitted to the neurological emergency room as candidates for intravenous thrombolysis for acute stroke.
\end{abstract}

\section{Case 1}

An elderly woman with a history of high blood pressure and hypercholesterolemia was admitted to the emergency room for suspected stroke due to abruptly starting vertigo, blurred vision and difficulties in speech. Neurological examination within $3 \mathrm{~h}$ from the symptom onset revealed high blood pressure (206/130 mm Hg), dysarthria and impaired right hand coordination. A CT scan showed no abnormalities, and CT angiography showed nonsignificant atherosclerotic changes. Minor cerebral infarction was suspected and clopidogrel treatment was started.

Twelve hours after symptoms onset, the patient could not speak or swallow but was able to move all extremities. The patient was intubated because of breathing difficulties and $\mathrm{CO}_{2}$ retention. At $24 \mathrm{~h}$, all cranial nerve functions were absent. The patient could not open her eyes, here pupils were dilated, and there were no spontaneous respiration or movements. Yet, the patient was able to communicate with 'yes' and 'no' answers by a weak squeeze of the right hand. A brain MRI revealed an old hemorrhage in the pons, and CSF findings were normal. Nerve conduction study findings showed that motor amplitudes were negligible, but sensory responses were preserved. In electromyography of the finger flexor muscles, pronounced jiggle (i.e. jitter with concentric needle) (fig. 1) was observed, but no increment was found in repeated nerve stimulation (RNS). These findings, together with the clinical findings, led to suspicion of botulism. 
Serum, gastric fluid and stool samples were taken, and botulinum antitoxin was given 3 days after symptom onset. Despite all treatment efforts, the patient died 13 days after admission to the hospital due to septic shock and multiorgan failure.

\section{Case 2}

Three days after the arrival of the first patient, a previously healthy 29-year-old woman was admitted to the emergency room for suspected stroke. The patient had woken up with dizziness, diplopia and difficulties in swallowing. Neurological examination revealed dysphasia, diplopia and absent gag reflex. The findings of MRI and MRA were normal. It was soon found that the patient was a relative of patient 1 , and that they had eaten the same food. Although she received the antitoxin treatment the same evening, her neurological symptoms deteriorated during the first 2 days. She needed assisted ventilation for 3 weeks, although other symptoms were resolved within 1 week.

The common foodstuff eaten by both patients were Italian olives. Those olives were strongly positive for botulinum neurotoxin type $B$, and the gastric content and fecal samples of both patients tested by multiplex PCR were positive for Clostridium botulinum type B. In addition, the serum of patient 1 was weakly positive for botulinum neurotoxin [1]

\section{Discussion}

Botulinum toxin binds irreversibly to presynaptic plates of the neuromuscular junction causing dysfunction of voluntary muscles, inner organs and the autonomic nervous system. In addition, the toxin may modify the inhibition in the central nervous system, possibly due to altered afferent input from the muscle spindles [2].

Botulinum antitoxin neutralizes only the circulating toxin and should thus be administrated as soon as possible before completing the laboratory tests. The diagnosis must therefore be based on clinical findings; bulbar palsy with descending paralysis is the most typical finding. Especially the combination of bilateral drooping of the eye lids, absent gag reflex and respiratory failure with relatively well preserved limb forces and a clear mental status are suggestive of botulism and, more uncommonly, of other possible diagnoses like brainstem ischemia or polyradiculitis. Botulism diagnosis is further supported by normal MRI and CSF findings. According to the literature, as a sign of presynaptic transmission defect, high-frequency RNS shows often an incremental motor response in botulism patients. However, no increment was observed in our two cases, possibly due to largely destroyed endplates.

Neurological symptoms may develop acutely in food-borne botulism and may therefore be misinterpreted as brainstem ischemia. This may delay antitoxin treatment and worsen the prognosis of the patient.

\section{Disclosure Statement}

None of the authors have a conflict of interest to declare. 
a

a
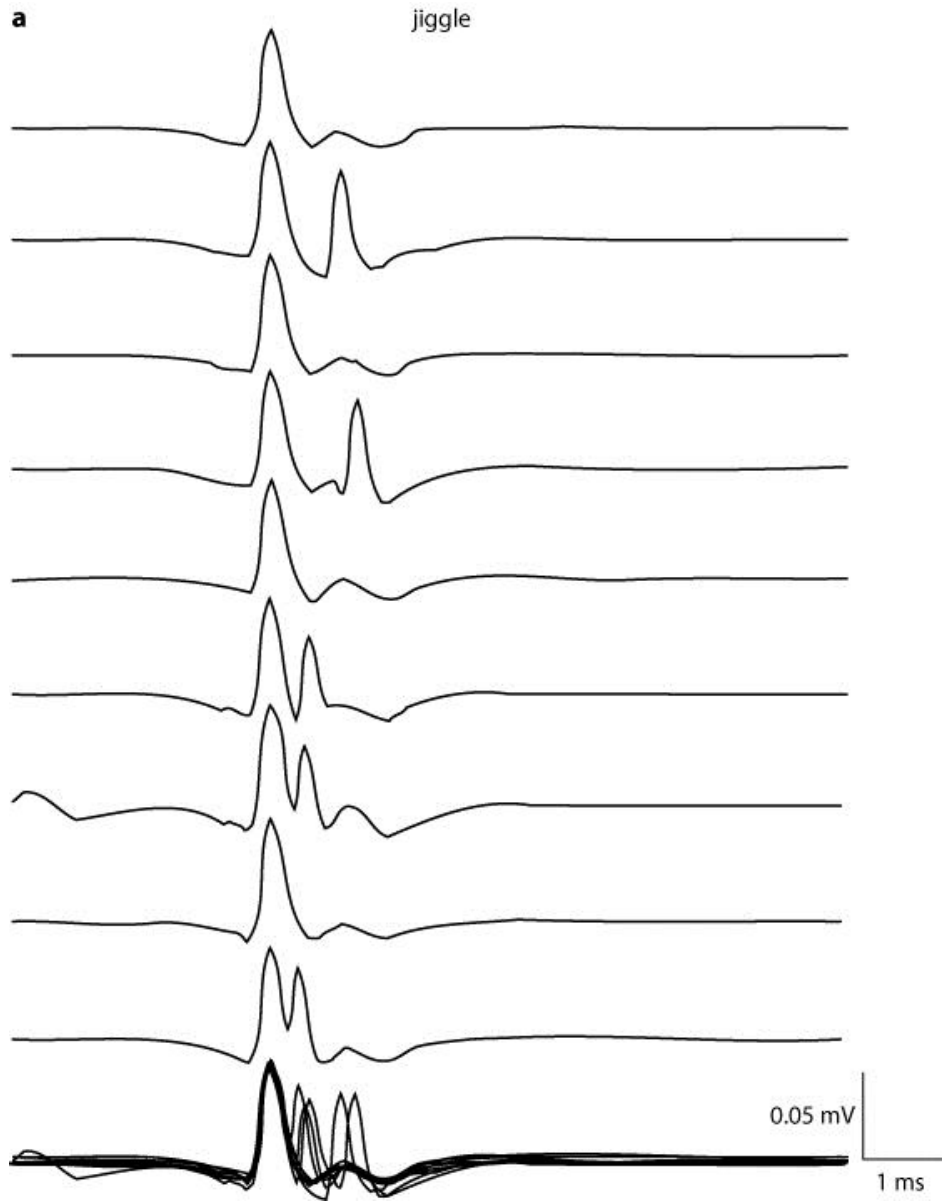

RNS

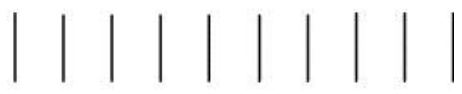

$3 \mathrm{~Hz}, 3.8 \mathrm{mV}:-3 /-3 \%$

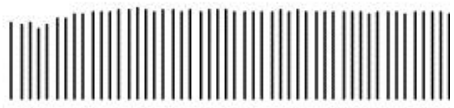

$30 \mathrm{~Hz}, 4.2 \mathrm{mV}: 11 /-16 \%$

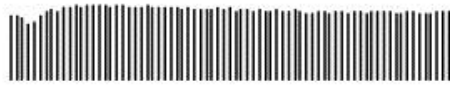

$40 \mathrm{~Hz}, 3.6 \mathrm{mV}:-6 /-9 \%$

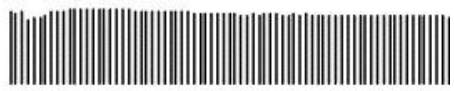

$50 \mathrm{~Hz}, 4 \mathrm{mV}:-6 /-20 \%$

Fig. 1. Jiggle in electromyography curves of the deep finger flexor muscle (a) and curves of RNS of the abductor digiti minimi muscle in patient 2 (b).

\section{References}

1 Jalava K, Selby K, Pihlajasaari A, Kolho E, Dahlsten E, Forss N, Bäcklund T, Korkeala H,

Honkanen-Buzalski T, Hulkko T, Derman Y, Järvinen A, Kotilainen H, Kultanen L,

Ruutu P, Lyytikaïnen 0, Lindström M: Two cases of food-borne botulism in Finland caused by conserved olives. Euro Surveill 2011;16:20034.

2 Hallet M: How does botulinum toxin work? Ann Neurol 2000;48:7-8. 